\title{
¿La colecistogastrostomía guiada por ultrasonido endoscópico es suficiente para resolver la obstrucción biliar distal maligna por cáncer pancreático?
}

\section{Is endoscopic ultrasound-assisted cholecystogastrostomy sufficient to resolve malignant distal biliary obstruction from pancreatic cancer?}

\author{
Gabriel Mosquera-Klinger, ${ }^{{ }^{*}}$ (D) Álvaro Andrés Gómez-Venegas, ${ }^{1}$ (1) Jhon Jaime Carvajal-Gutierrez. ${ }^{1}$ (1)
}

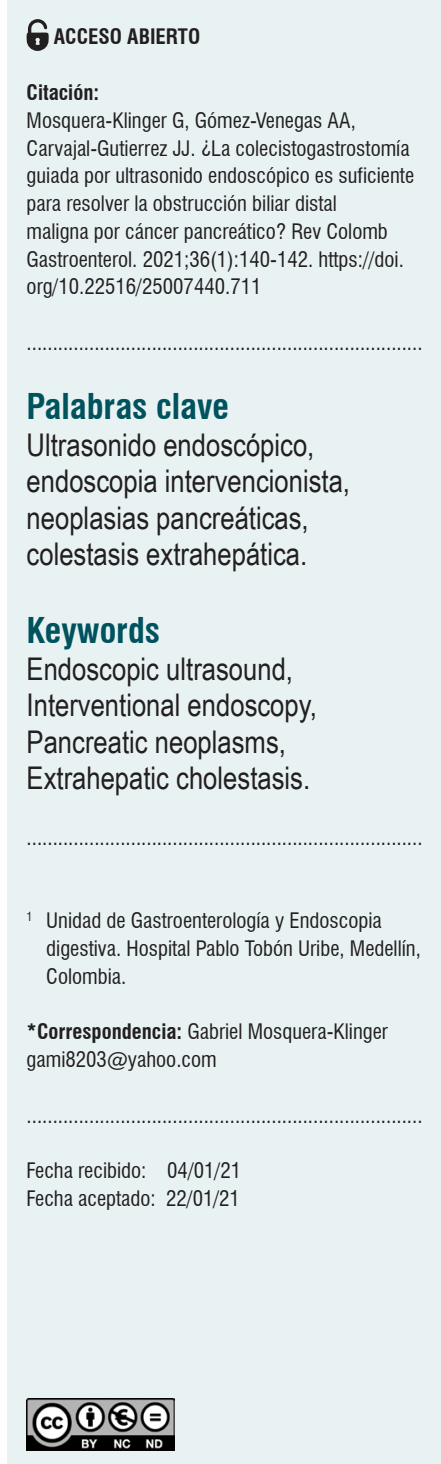

Señor editor:

De antemano felicitamos a el Dr. Pinto y colaboradores por la presentación de un caso realizado en Colombia, en el que es evidente el éxito técnico, en un procedimiento elegante y que requiere alta experticia en ultrasonido endoscópico (1).

En la actualidad, la colangiopancreatografía retrógrada endoscópica (CPRE) es el estándar de tratamiento para la ictericia por obstrucción biliar distal maligna en pacientes con enfermedad avanzada o con fines paliativos $(2,3)$. En los casos en que la CPRE no sea factible o sea fallida, sin lograr el acceso a la papila, el drenaje biliar guiado por ultrasonografía endoscópica (USE-guiada) se considera un tratamiento alternativo efectivo (3-5). La coledocoduodenostomía USE-guiada es la técnica endoscópica preferida por muchos autores cuando la obstrucción biliar es distal y la hepaticogastrostomía es preferida en casos de obstrucción biliar proximal o cuando hay falta de acceso por el duodeno $(3,4)$.

En los casos en que la CPRE sea fallida y no sea posible realizar una derivación biliar USE-guiada, podría ser más eficiente y segura una derivación biliar transparietohepática (derivación percutánea). La mayoría de instituciones de alta complejidad cuentan con radiología intervencionista y, en términos de frecuencia, la derivación transparietohepática suele ser más común, por lo que podría existir mayor familiaridad con esta, dato no menor ya que esto podría disminuir los riesgos relacionados con el procedimiento.

En un estudio realizado en 2 centros universitarios de un país latinoamericano, que fue publicado recientemente, se describió la experiencia en la derivación transparietohepática en estenosis obstructiva de la vía biliar. Con una muestra de más de 500 pacientes, la mayoría de casos se debió a obstrucción biliar maligna y en más de 70,4\% de los casos la patencia del catéter fue igual o mayor a 6 meses; en este se presentaron complicaciones (hemobilia e infección) en 12,2\% de los casos (6).

En los casos de obstrucción biliar, el drenaje de la vesícula podría impactar de forma residual sobre la carga de bilis en el ducto biliar principal, en especial, porque las válvulas de Heister del conducto cístico tienen entre sus funciones evitar el flujo retrógrado biliar desde el colédoco hasta la vesícula (7). En los casos referenciados en el artículo del Dr. Pinto y colaboradores, el drenaje de la vesícula se realizó en un caso después de haber intentado una hepatogastrostomía. En este, después de haber puncionado y dibujado el árbol biliar se determinó que no era factible derivarlo por esa vía (8). En el resto de casos 
referenciados, el drenaje de la vesícula USE-guiada fue en el escenario de colecistitis aguda, no ocurrió específicamente por la obstrucción biliar (9-13).

Hasta el momento se conoce solo un estudio retrospectivo, con 12 individuos sometidos a drenaje de vesícula USE-guiada con el propósito de derivar la vía biliar en pacientes con obstrucción biliar maligna. En este trabajo, se realizó el drenaje de la vesícula USE-guiado como rescate en el intento de drenar la vía biliar en individuos en quienes el drenaje biliar USE-guiada no fue posible y rechazaron el drenaje percutáneo porque querían evitar el tubo de drenaje externo. En ese estudio se describió el éxito técnico en todos los casos y el éxito funcional en el $91 \%$ de casos (definieron el éxito funcional en la reducción de más del $50 \%$ de la bilirrubina total en el seguimiento a 2 semanas); aunque se presentaron complicaciones en el $16,9 \%$ de casos (14).

Es posible que a pesar de que la vesícula sea una diana grande y muchas veces más expedita para puncionar y drenar, no siempre se logre el objetivo de derivar de forma efectiva la vía biliar en obstrucciones biliares malignas.

En este caso en particular, en el que se evidenció una vesícula y un conducto cístico tan dilatado, podría ser de interés como complemento al drenaje biliar pasar un balón de Fogarty a través de la cistogastrostomía para ocluirla; luego, aplicar medio de contraste para realizar una colangiografía por vía transcística; posteriormente, pasar una guía hacia la vía biliar y, finalmente, utilizar esa comunicación gastrocística para drenar la vía biliar con un stent doble cola de cerdo. Otra opción plausible podría ser el paso de un endoscopio convencional a través de la cistogastrostomía, avanzar una guía de forma directa al colédoco y drenar la vía biliar con un stent doble cola de cerdo.

En el intervencionismo endoscópico se debe tener mucha claridad de los objetivos y de la diana a utilizar para el abordaje. Por una parte, en casos de colecistitis, vesícula hidrópica que produzca síntomas en pacientes no candidatos quirúrgicos o pacientes que rechacen la derivación percutánea se debería intentar el drenaje USE-guiada de la vesícula; pero si el problema clínico es la obstrucción biliar, la diana debería ser el conducto biliar (ya sea USE-guiada o por vía transparietohepática) como primera instancia. Por otra parte, en los casos con obstrucción duodenal, en los que el duodenoscopio no logre sobrepasar la estenosis duodenal (como en este caso descrito), se debería intentar resolver la obstrucción del tracto de salida mediante prótesis duodenal o una gastroyeyunostomía USE-guiada para paliar de forma completa los problemas clínicos del paciente.

En conclusión, la cistogastrostomía USE-guiada es una excelente opción en el escenario de colecistitis aguda o vesícula hidrópica sintomática en pacientes que no sean candidatos quirúrgicos. Es posible que los beneficios en el drenaje biliar mediante una cistogastrostomía sean limitados.

En casos de obstrucción biliar distal maligna en los que no sea factible la CPRE ni el drenaje biliar USE-guiada, podría ser más efectiva y segura la opción de una derivación biliar transparietohepática; pero en los casos en los que no sea factible o sea rechazada la derivación biliar transparietohepática, entonces se podría ofrecer la derivación USE-guiada de la vesícula como un método compasivo de rescate.

\section{REFERENCIAS}

1. Pinto R, Solano J, Cabrera LF, Benito E.

Colecistogastrostomía guiada por ultrasonido endoscópico en un paciente con cáncer pancreático: primer caso en Colombia (con video). Rev Colomb Gastroenterol. 2020;35(4):527-532. https://doi. org/10.22516/25007440.400

2. Forero EA, Galasso D, Bories E, Giovannini M. Derivaciones biliopancreáticas guiadas por ultrasonido endoscópico: descripción de caso y revisión de la literatura. Rev Colomb Gastroenterol. 2013;28(4):339-351.

3. Minaga K, Takenaka M, Yamao K, Kamata K, Omoto S, Nakai A, Yamazaki T, Okamoto A, Ishikawa R, Yoshikawa T, Chiba Y, Watanabe T, Kudo M. Clinical utility of treatment method conversion during single-session endoscopic ultrasound-guided biliary drainage. World J Gastroenterol. 2020;26(9):947-959. https://doi. org/10.3748/wjg.v26.i9.947
4. Perez-Miranda M, de la Serna C, Diez-Redondo P, Vila JJ. Endosonography-guided cholangiopancreatography as a salvage drainage procedure for obstructed biliary and pancreatic ducts. World J Gastrointest Endosc. 2010;2(6):21222. https://doi.org/10.4253/wjge.v2.i6.212

5. de Benito Sanz M, Nájera-Muñoz R, de la Serna-Higuera C, Fuentes-Valenzuela E, Fanjul I, Chavarría C, GarcíaAlonso FJ, Sanchez-Ocana R, Carbajo AY, Bazaga S, PerezMiranda M. Lumen apposing metal stents versus tubular self-expandable metal stents for endoscopic ultrasoundguided choledochoduodenostomy in malignant biliary obstruction. Surg Endosc. 2020. https://doi.org/10.1007/ s00464-020-08179-y

6. Muñoz R, Salazar G, Velásquez J, Prasca M, Lander B. Drenaje biliar transparietohepático: Experiencia en el hospital universitario y centro médico de Caracas. Rev Colomb Radiol. 2018;29(3):4957-62. 
7. Dasgupta D, Stringer MD. Cystic duct and Heister's “valves". Clin Anat. 2005;18(2):81-7. https://doi. org/10.1002/ca.20118

8. Itoi T, Binmoeller K, Itokawa F, Umeda J, Tanaka R. Endoscopic ultrasonography-guided cholecystogastrostomy using a lumen-apposing metal stent as an alternative to extrahepatic bile duct drainage in pancreatic cancer with duodenal invasion. Dig Endosc. 2013;25 Suppl 2:137-41. https://doi.org/10.1111/den.12084

9. Itoi T, Coelho-Prabhu N, Baron TH. Endoscopic gallbladder drainage for management of acute cholecystitis. Gastrointest Endosc. 2010;71(6):1038-45. https://doi. org/10.1016/j.gie.2010.01.026

10. Itoi $T$, Isayama $H$, Sofuni $A$, Itokawa $F$, Kurihara $T$, Tsuchiya T, Tsuji S, Ishii K, Ikeuchi N, Tanaka R, Umeda J, Moriyasu F, Kawakami H. Stent selection and tips on placement technique of EUS-guided biliary drainage: transduodenal and transgastric stenting. J Hepatobiliary Pancreat Sci. 2011;18(5):664-72. https://doi.org/10.1007/s00534011-0410-9

11. Jang JW, Lee SS, Park DH, Seo DW, Lee SK, Kim MH. Feasibility and safety of EUS-guided transgastric/trans- duodenal gallbladder drainage with single-step placement of a modified covered self-expandable metal stent in patients unsuitable for cholecystectomy. Gastrointest Endosc. 2011;74(1):176-81. https://doi.org/10.1016/j. gie.2011.03.1120

12. Súbtil JC, Betes M, Muñoz-Navas M. Gallbladder drainage guided by endoscopic ultrasound. World J Gastrointest Endosc. 2010;2(6):203-9. https://doi.org/10.4253/wjge. v2.i6.203

13. Widmer J, Alvarez P, Gaidhane M, Paddu N, Umrania H, Sharaiha R, Kahaleh M. Endoscopic ultrasonographyguided cholecystogastrostomy in patients with unresectable pancreatic cancer using anti-migratory metal stents: a new approach. Dig Endosc. 2014;26(4):599-602. https: //doi. org/10.1111/den.12163

14. Imai H, Kitano M, Omoto S, Kadosaka K, Kamata K, Miyata T, Yamao K, Sakamoto H, Harwani Y, Kudo M. EUS-guided gallbladder drainage for rescue treatment of malignant distal biliary obstruction after unsuccessful ERCP. Gastrointest Endosc. 2016;84(1):147-51. https:// doi.org/10.1016/j.gie.2015.12.024 